\section{Proven alternatives}

In light of the European Parliament's vote to phase down amalgam, it has become more important than ever for dentists to find trustworthy alternatives. This means you need new restorative materials you can trust to deliver the same easy handling, durability and effectiveness, without any of the negative side effects. $3 \mathrm{M}$ Oral Care offers several products that are more than capable of rising to this challenge.

Among these is the Filtek One bulk fill restorative (Fig. 1), which enables clinicians to create excellent restorations ${ }^{1}$ with speed and simplicity. ${ }^{2}$ It allows for easy sculpting and shaping, ${ }^{3}$ with good adaptability to the cavity and margins, ${ }^{4}$ low shrinkage when cured ${ }^{5}$ and slump resistance for simple placement. ${ }^{6}$ Improving the professional workflow, one-step placement of the bulk fill material saves precious time $e^{7}$ compared to traditional composite procedures.

In terms of aesthetics, the Filtek One material is available in shades that match the demands for posterior restorations, ${ }^{8}$ enabling the fabrication of natural-looking outcomes. ${ }^{9}$ What's more, Filtek One bulk fill restorative features higher wear resistance, ${ }^{10}$ compressive ${ }^{11}$ and flexural strength, ${ }^{12}$ polish retention ${ }^{13}$ and fracture toughness ${ }^{14}$ than other leading brands.

\section{Dr Sharon Caro, Principal of St Annes Private Dental Centre in} Lancashire, said:

'Filtek One's handling properties are excellent; it doesn't stick and it's very easy to manipulate. The aesthetics are absolutely fantastic; the colours are very good and the translucency is very similar to the tooth tissue, providing highly natural-looking restorations, as can be seen in the photographs.

'The material also doesn't require layering for beautiful aesthetics, therefore there is no need to use clear or enamel coloured surface product and it is incredibly easy to finish - polishing is quick and easy with the Sof Lex discs. This saves a huge amount of time.

'I would definitely recommend Filtek One to other practitioners.'

Ketac Universal glass ionomer restorative is another effective alternative to amalgam (Fig. 2). With an ideal viscosity ${ }^{15}$ and a less sticky consistency in comparison to other glass-ionomer solutions, ${ }^{16}$ it allows for easy application. In fact, $78 \%$ of dentists who trialled the product reported excellent overall handling $(\mathrm{n}=51) .{ }^{17}$ To complement this and further encourage a simple treatment procedure, ${ }^{18}$ Ketac Universal requires no layering, ${ }^{19}$ conditioner or coating. ${ }^{20}$ The fewer steps afford a smoother workflow and reduced chair time ${ }^{21}$ for increased patient comfort.

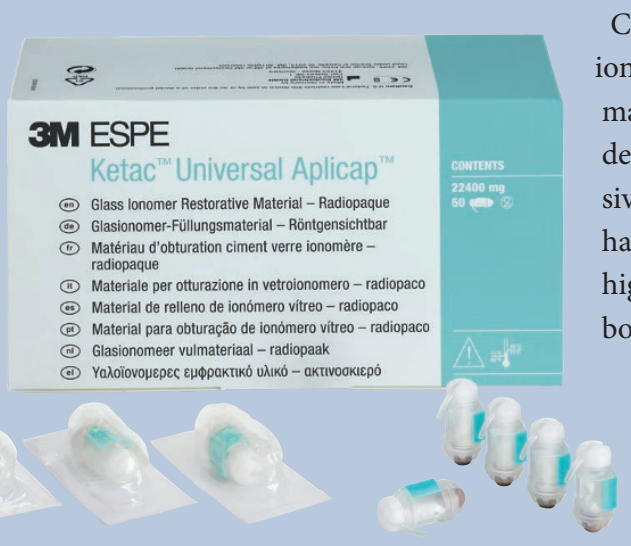

Compared to other glassionomer materials on the market, Ketac Universal delivers higher compressive strength ${ }^{22}$ and surface hardness. ${ }^{23}$ It also features high wear resistance ${ }^{24}$ for both stress bearing and nonstress bearing restorations, ${ }^{25}$ giving you and your patients confidence that long-lasting results will be achieved. ${ }^{26}$
An additional benefit of Ketac Universal glass-ionomer restorative is that it continuously releases more fluoride than competitive

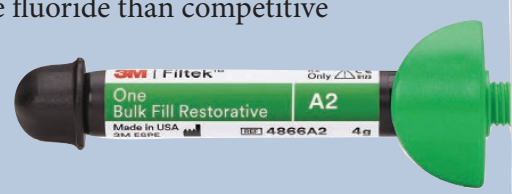

Fig. $13 \mathrm{M}^{\mathrm{TM}}$ Filtek $^{\mathrm{TM}}$ One Bulk Fill Restorative

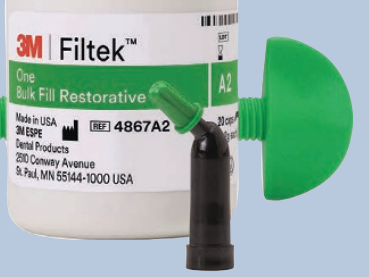

products ${ }^{27}$ for at least 12 months post placement. ${ }^{28}$ As fluoride has been shown to aid in caries prevention, ${ }^{29,30}$ this is a great advantage for any patients who experience recurrent caries.

Dr Kunal Shah, Principal of LeoDental in London, commented:

'I think Ketac Universal is a fantastic product - it does everything I need it to do. It doesn't stick and is one of the easiest products on the market to place. It's strong, quick and easy to use, with a simple workflow that promotes patient comfort. The steady release of fluoride is also a benefit for maintaining patients' general oral health.

'Although I would rarely give a product 10 out of 10, I think Ketac Universal deserves it! I love it - it's my go-to product for core build ups.'

If you're looking for materials to replace amalgam, you need look no further than the clinically proven portfolio from $3 \mathrm{M}$ Oral Care.

For more information, call 0800626578 or visit www.3M.co.uk/Dental

\section{$3 M$, Filtek and Ketac are trademarks of the $3 M$ Company.}

1. 3M Oral Care Data. Filtek One. Excellent restorations. Claim 6783, 2016 2. 3M Oral Care Data. Filtek One. Speed and simplicity. Claim 6065, 2014.

3. 3M Oral Care Data. Filtek One. Sculpting and shaping. Claim 6761, 2016.

3M Oral Care Data. Filtek One. Adaptability. Claim 6763, 2016. 3M Oral Care Data. Filtek One. Low shrinkage. Claim 6823, 2016.

6. 3M Oral Care Data. Filtek One. Slump resistance. Claim 6771, 2016. 3M Oral Care Data. Filtek One. Time saving. Claim 6781, 2016.

3M Oral Care Data. Filtek One. Shade matching. Claim 6065, 2016. 3M Oral Care Data. Filtek One. Natural looking. Claim 6786, 2016.

10. 3M Oral Care Data. Filtek One. Wear resistance. Claim 6863, 2016.

11. 3M Oral Care Data. Filtek One. Compressive strength. Claim 6841, 2016

12. 3M Oral Care Data. Filtek One. Flexural strength. Claim 6842, 2016.

13. 3M Oral Care Data. Filtek One. Polish retention. Claim 6839, 2016.

14. 3M Oral Care Data. Filtek One. Fracture toughness. Claim 6840, 2016.

15. 3M Oral Care Data. Ketac Universal. Ideal viscosity. Claim 6227, 2014.

16. 3M Oral Care Data. Ketac Universal. Less sticky. Claim 6357, 2014

17. 3M Oral Care Data. Ketac Universal. Excellent overall handling. Claim 6243, 2014

18. 3M Oral Care Data. Ketac Universal. Fewer steps = simpler. Claim 6333, 2014.

19. 3M Oral Care Data. Ketac Universal. Bulk fill. Claim 6175, 2014.

20. 3M Oral Care Data. Ketac Universal. No conditioner or coating needed. Claim 6331, 2014

21. 3M Oral care data. Ketac Universal. Reduced chair time. Claim 6332, 2014.

22. 3M Oral Care Data. Ketac Universal. Compressive strength. Claim 6178, 2014

23. 3M Oral Care Data. Ketac Universal. Higher surface hardness. Claim 6251, 2014

24. 3M Oral Care Data. Ketac Universal. Wear resistance. Claim 6180, 2014.

25. 3M Oral Care Data. Ketac Universal. Reliable choice in non- stress bearing and stress bearing restorations. Claim 6185, 2014

26. 3M Oral Care Data. In an application test where 3500 fillings were placed, $61 \%$ of the dentists were confident to use Ketac Universal for long-term restorations (more than two years). Claim 6237, 2014

27. 3M Oral Care Data. Ketac Universal. Higher fluoride release. Claim 6253, 2014.

28. 3M Oral Care Data. Ketac Universal. Continuous fluoride release. Claim 6177, 2014.

29. Ditmyer M, Dounis G, Mobley C, Schwarz E. A case-control study of determinants for high and low dental caries prevalence in Nevada youth. BMC Oral Health 2010; DOI: 10.1186/1472-6831-10-24

30. Griffin S O, Regnier E, Griffin P M, Huntley V. Effectiveness of fluoride in preventing caries in adults. J Dent Res 2007; 86: 410-415. 